\title{
The Evolution of Trypanosomes Infecting Humans and Primates
}

\author{
Jamie Stevens $/{ }^{+}$, Harry Noyes*, Wendy Gibson
}

\author{
School of Biological Sciences, University of Bristol, Bristol BS8 1UG, UK *Liverpool School of Tropical \\ Medicine, Pembroke Place, Liverpool L3 5QA, UK
}

\begin{abstract}
Based on phylogenetic analysis of $18 S$ rRNA sequences and clade taxon composition, this paper adopts a biogeographical approach to understanding the evolutionary relationships of the human and primate infective trypanosomes, Trypanosoma cruzi, T. brucei, T. rangeli and T. cyclops. Results indicate that these parasites have divergent origins and fundamentally different patterns of evolution. T. cruzi is placed in a clade with $\mathrm{T}$. rangeli and trypanosomes specific to bats and a kangaroo. The predominantly South American and Australian origins of parasites within this clade suggest an ancient southern super-continent origin for ancestral T. cruzi, possibly in marsupials. T. brucei clusters exclusively with mammalian, salivarian trypanosomes of African origin, suggesting an evolutionary history confined to Africa, while T. cyclops, from an Asian primate appears to have evolved separately and is placed in a clade with $\mathrm{T}$. (Megatrypanum) species. Relating clade taxon composition to palaeogeographic evidence, the divergence of $\mathrm{T}$. brucei and $\mathrm{T}$. cruzi can be dated to the mid-Cretaceous, around 100 million years before present, following the separation of Africa, South America and Euramerica. Such an estimate of divergence time is considerably more recent than those of most previous studies based on molecular clock methods. Perhaps significantly, Salivarian trypanosomes appear, from these data, to be evolving several times faster than Schizotrypanum species, a factor which may have contributed to previous anomalous estimates of divergence times.
\end{abstract}

Key words: Trypanosoma brucei - Trypanosoma cruzi - evolution - phylogenetics - small subunit ribosomal RNA - biogeography

The evolutionary relationships of human infective trypanosomes have long been debated (Baker 1963, Hoare 1972, Vickerman 1994). However, it is only recently, with advances in molecular and phylogenetic methods, that significant progress towards resolving trypanosome evolutionary history is being made (e.g. Lake et al. 1988, Fernandes et al. 1993, Wiemer et al. 1995, Maslov et al. 1996, Lukes et al. 1997, Haag et al. 1998, Stevens et al. 1998).

Prior to the advent of molecular techniques, great importance was attached to the mode of transmission as a means of understanding the evolutionary history of trypanosomes (Baker 1963, Hoare 1972). Most vertebrate trypanosomes are transmitted from host to host by bloodsucking arthropod or leech vectors. The trypanosomes are taken up by the vector with a bloodmeal, and usu-

\footnotetext{
This work was supported by the Wellcome Trust (Grant No. 047131/Z/96/Z).

${ }^{+}$Corresponding author. Fax: +44-117-925.7374. E-mail: j.r.stevens@bristol.ac.uk

Received 15 June 1998

Accepted 30 July 1998
}

ally undergo one or more cycles of development and multiplication in the alimentary tract of the invertebrate, before infective forms are transmitted to a new vertebrate host via saliva, contamination with faeces or ingestion of the whole vector. In this respect the human pathogenic trypanosomes differ markedly. T. brucei, the causative agent of African human sleeping sickness, together with a range of related species of veterinary importance (T. congolense, T. simiae and T. vivax), is transmitted by tsetse flies (genus Glossina) by the salivarian route. T. cruzi, which causes Chagas disease in Latin America, develops in the hindgut of triatomine bugs; infective forms are excreted in the faeces and infect a new host by contamination of wounds or mucous membranes - the stercorarian route (Hoare 1972). The classification and transmission characteristics of another, apparently nonpathogenic species from Latin America, T. rangeli, remain much in debate.

In addition to transmission characteristics, $T$. bruce $i$ and T. cruzi also differ in their mode of infection: T. brucei resides in the bloodstream and evades the host immune response by antigenic variation, while $T$. cruzi is an intracellular parasite and multiplies in tissue pseudocysts, with a tran- 
sient bloodstream phase in the host. It has thus been obvious from even the earliest parasitological studies that $T$. bruce $i$ and $T$. cruzi are very different organisms, but, just how different? Such a question has an important bearing on how far results relating to the biochemistry or metabolism of one pathogenic species can be extrapolated to the other, for example, in terms of new chemotherapeutic approaches.

To quantify the evolutionary distance between the two species we have used the divergence of the small subunit ribosomal RNA (ssu rRNA) gene to date the evolutionary split (Stevens et al. 1998). Similar molecular phylogenetic studies have previously relied heavily on "molecular clocks", calibrated by a variety of methods (e.g. Lake et al. 1988, Fernandes et al. 1993, Haag et al. 1998). However, given the almost constant debate surrounding the accuracy of such clocks (Sibley \& Ahlquist 1984, Wilson et al. 1987), we have based the date of divergence of T. brucei and T. cruzi on biogeographical and clade taxon composition (Nelson \& Rosen 1981, Meyers \& Giller 1988). We believe that this approach is more likely to yield a phylogenetic interpretation with biological relevance, which will contribute to an understanding of the evolution of the genus Trypanosoma. Finally, the addition of more taxa, including a primate trypanosome from south-east Asia, T. cyclops, has allowed us to explore the robustness of the phylogeny and our biogeographically based evolutionary hypotheses.

\section{MATERIALS AND METHODS}

Choice of phylogenetic marker - The $18 \mathrm{~S}$ ssu rRNA gene was chosen as a suitable phylogenetic marker. It is conserved throughout the eukaryotes, while the range of conserved and variable regions allow diverse rates of genetic evolution to be studied, making it ideal for elucidating both higher evolutionary relationships and those between closely related species (Sogin et al. 1986). Its high copy number also facilitates ease of PCR amplification. The ssu rRNA gene has become the marker of choice for evolutionary analyses of the kinetoplastid protozoa (e.g. Fernandes et al. 1993, Maslov et al. 1994, 1996, Marché et al. 1995, Lukes et al. 1997, Haag et al. 1998).

Trypanosomes - Summary details of all taxa are given in the Table. T. cyclops was isolated from Macaca spp. in Peninsular Malaysia (Weinman 1972). This uniquely pigmented trypanosome (described as containing large granules of pigment derived from haemoglobin) could not readily be placed in any existing subgenus. The vector is unknown, but transmission by reduviid bugs was ruled out (Weinman 1972).
Ribosomal RNA sequences - The ssu rRNA sequence of $T$. cyclops was sequenced, as described by Stevens et al. (1998). Briefly, the gene was amplified by PCR from trypanosome template DNA as a fragment of $\sim 2 \mathrm{~kb}$ using conserved primers (Maslov et al. 1996). The products of 8-10 separate PCR reactions were then purified and pooled, prior to automated sequencing in both directions at approximately 300 base pair intervals using 12 additional internal primers (Maslov et al. 1996) on a Perkin-Elmer ABI 377 automated sequencer. A consensus sequence was assembled for each trypanosome strain from the internal primer sequences using AutoAssembler v.2.0 (Applied Biosystems, Perkin-Elmer).

Thirty-two Trypanosoma sp. sequences were included from Stevens et al. (1998; Table), together with 15 Trypanosoma sp. sequences from the EMBL/GenBank databases. The suitability of freeliving bodonid taxa as outgroups for phylogenetic analysis of trypanosomatids has been established by a number of studies using a range of ribosomal and protein coding genes (see Stevens et al. 1998). In this study, Trypanosoma species were compared with a range of ten outgroup taxa (Bodo caudatus X53910; Trypanoplasma borreli L14840; Crithidia spp. X03450, L29264; Leishmania spp. X53912, X07773, X53913, X53915; and Phytomonas spp. L35076, L35077).

Alignments - The T. cyclops sequence was incorporated into the alignment of Stevens et al. (1998). In this alignment, all sequences were aligned primarily to eight Trypanosoma sequences downloaded from the rRNA database maintained at the University of Antwerp (Neefs et al. 1990); the alignment of these eight template sequences is based on their secondary structure. Sub-sections of the alignment, between 'anchor' regions of high homology were then sub-aligned using the program Clustal V (Higgins et al. 1992), before final adjustments were made by eye. Hypervariable sites, where nucleotide changes were saturated, and regions where it was not possible to produce a single reliable alignment across all 58 taxa were excluded from the analysis. Following this, a number of separate alignments, representing more or less stringent subsets of a 'standard' alignment, were explored (Stevens et al. 1998) and used as the basis for the phylogenetic analysis presented in this paper. Certain sites which were locally informative between closely related taxa introduced 'noise', resulting in a loss of definition (reduced bootstrap support) at higher phylogenetic levels; such sites were excluded from the final analysis (Fig.) and the alignment used included 1801 nucleotide positions (available on request from JRS).

Phylogenetic analyses - Bootstrapped maxi- 
TABLE

Summary details of Trypanosoma spp. analysed ${ }^{a}$

\begin{tabular}{|c|c|c|c|}
\hline Species & Host & & Location \\
\hline T. avium & Bird & Fringilla coelebs & Czech Republic \\
\hline T. brucei gambiense & Human & Homo sapiens & Nigeria \\
\hline T. brucei rhodesiense & Human & Homo sapiens & Uganda \\
\hline T. cobitis & Freshwater fish & Noemacheilus barbatulus & England \\
\hline T. congolense (kilifi) & Domestic goat & Capra sp. & Kenya \\
\hline T. congolense (forest & Domestic goat & Capra sp. & Cameroon \\
\hline T. congolense (savannah) & Domestic goat & Capra sp. & Kenya \\
\hline T. cruzi (Z I) & Human & Homo sapiens & Brazil \\
\hline T. cruzi (Z II) & Triatomine bug & Triatoma infestans & Chile \\
\hline T. cruzi (Z III) & Human & Homo sapiens & Brazil \\
\hline T. cruzi marinkellei & Bat & Phyllostomum discolor & Brazil \\
\hline T. cyclops & Macaque & Macaca sp. & Malaysia \\
\hline T. dionisii & Bat & Pipistrellus pipistrellus & England \\
\hline T. dionisii & Bat & Pipistrellus pipistrellus & Belgium \\
\hline T. equiperdum & Horse & Equus caballus & China \\
\hline T. evansi & Capybara & H. hydrochaeris & Brazil \\
\hline T. godfreyi & Tsetse fly & G.m.submorsitans & The Gambia \\
\hline T. grayi & Tsetse fly & G.p.gambiensis & The Gambia \\
\hline T. lewisi & Rat & Rattus sp. & England \\
\hline T. mega & Toad & Bufo regularis & Africa \\
\hline T. microti & Vole & Microtis agrestis & England \\
\hline T. pestanai & Badger & Meles meles & France \\
\hline T. rangeli & Dog & Canis sp. & Venezuela \\
\hline T. rotatorium & Frog & Rana catesbeiana & Canada \\
\hline T. simiae & Tsetse fly & G.m.submorsitans & The Gambia \\
\hline T. theileri & Cattle & Bos taurus & Germany \\
\hline T. theileri & Cattle & Bos taurus & Scotland \\
\hline T. varani & Lizard & Varanus exanthematicus & Senegal \\
\hline T. vespertilionis & Bat & Pipistrellus pipistrellus & England \\
\hline$T$. sp. & Leech & Piscicola geometra & England \\
\hline T. sp. & Kangaroo & Macropus giganteus & Australia \\
\hline T. sp. & Wombat & Vombatus ursinus & Australia \\
\hline T. sp. & Deer & Cervus dama & Germany \\
\hline
\end{tabular}

$a$ : details of the 33 Trypanosoma sp. isolates sequenced as part of this study. See Stevens et al. (1998) for additional isolation details; see Weinman (1972) for full details of T. cyclops. Information on the 15 additional Trypanosoma sp. ssu rRNA sequences added from EMBL/GenBank can be obtained from database: T. boissoni U39580; T. carassii L14841; T. rotatorium U39583; T. triglae U39584; T. brucei brucei M12676; T. congolense (kilifi-type) U22317; $T$. congolense (forest-type) U22319; T. congolense (savannah-type) U22315; T. congolense (tsavo-type) U22318; T. simiae U22320; T. vivax U22316; T. cruzi X53917; T. cruzi M31432; T. avium U39578; T. scelopori U67182.

mum parsimony analysis of 58 kinetoplastid $18 \mathrm{~S}$ ssu rRNA sequences (Table) was performed with 100 replicates (Fig.); again, analyses were repeated with a number of more and less stringent alignments (see Stevens et al. 1998). The number of taxa necessitated the use of a heuristic search strategy to find the most parsimonious trees. The default options of PAUP were used: TBR branch swapping, zero length branches collapsed and 10 random addition sequences (bootstrap analyses used simple addition).

Maximum-likelihood analysis was also performed; however, due to computational constraints, a reduced data set of 36 taxa was employed. Taxa were selected for inclusion in the maximum likelihood analysis so as to maximise the degree of sequence variation analysed, highly homologous sequences being excluded; starting trees were derived by both parsimony and neighbour-joining. Transition/transversion ratios were estimated from the data in preliminary runs and then set for full analyses. All analyses were performed using test version $4.0 \mathrm{~d} 63$ of PAUP*, written by David L Swofford.

\section{RESULTS}

Phylogenetic analysis. The phylogram (Fig.) classifies the genus Trypanosoma into three major clades, including the brucei-clade and the cruzi- 


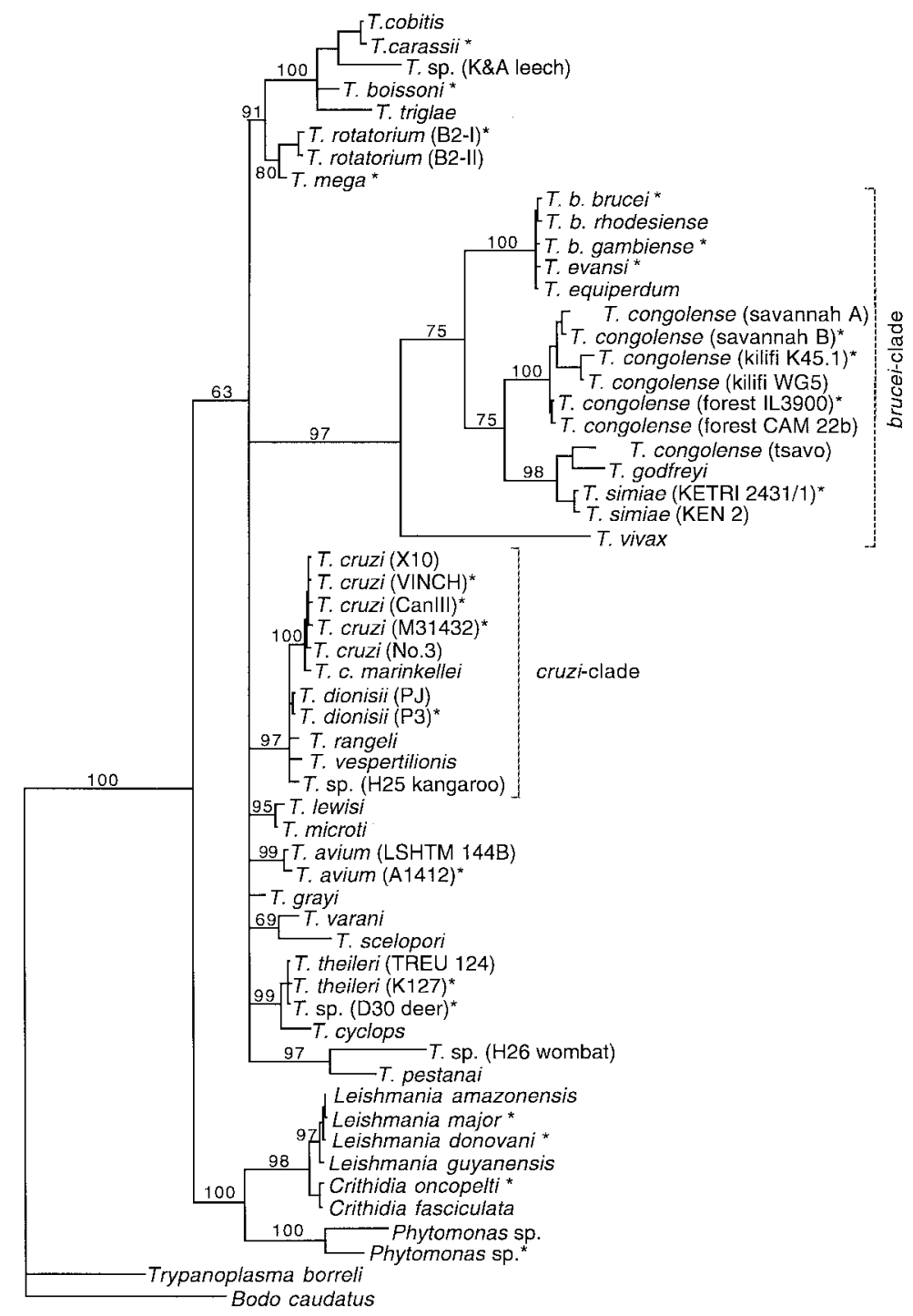

Phylogram constructed by bootstrapped (100 replicates) maximum parsimony analysis of 58 kinetoplastid 18S ssu rRNA sequences and rooted on the free-living kinetoplastid, Bodo caudatus. The tree is derived from the 28 most parsimonious trees of length $=1135(\mathrm{RI}=0.8177, \mathrm{CI}=0.5570)$, based an alignment of 1801 nucleotide sites. Bootstrap values for all major nodes are given and all branches receiving bootstrap support values $>50 \%$ are shown; relationships failing to achieve this level of support are shown as polytomies (i.e. branch points at which three or more branches arise from the ancestral line). Certain clades, referred to in the text, are defined by dashed brackets. The 22 taxa not included in the maximum-likelihood analyses are marked *. Details of all taxa are given in the Table.

clade [the brucei and cruzi clades (and all species therein) are defined in the Fig. The terms 'bruceiclade' and 'cruzi-clade' are used throughout this study to refer to the clades containing (a) all mammalian Salivarian (Hoare 1972) trypanosomes (brucei-clade) and (b) trypanosomes in the subgenus Schizotrypanum, plus T. rangeli and an as yet unidentified species of trypanosome from a kangaroo (cruzi-clade)], and six minor clades, which together form a nine-way polytomy. Importantly, the phylogram is proven to be robust and its structure is largely the same as that presented by (Stevens et al. 1998). However, the addition of two Phytomonas sp. to the analysis significantly reduces phylogenetic definition at the upper level of the Trypanosoma, such that the clade containing trypanosomes from aquatic and related hosts no longer diverges earlier than other Trypanosoma, and forms part of a nine-way polytomy within the genus. Such a result serves to underline the im- 
portant influence that the choice of outgroup taxa may exert on phylogenetic analyses.

T. cyclops, isolated from a Malaysian primate, is classified with $T$. theileri and another well characterized Megatrypanum species from a deer (Fig.; $100 \%$ bootstrap support), rather than in either the brucei-clade or the cruzi-clade, which contain all other human/primate infective trypanosomes [Johnson (1933) reported T. lewisi in the blood of a child, also in Malaysia]. Such a result does not support a close phylogenetic relationship with either African or South American human/primate infective trypanosomes, whilst its apparent lack of overt Megatrypanum characteristics (Weinman 1972) calls into question the taxonomic basis of the subgenus Megatrypanum.

The results of the parsimony analyses were again strongly supported by maximum-likelihood analysis. The positions and branching order of all major clades were identical between methods (irrespective of starting tree), and only minor variations in the positions of certain terminal taxa were apparent. Indeed, the main phylogenetic relationships revealed in the tree are as described by Stevens et al. (1998) and are largely robust to the addition of the primate trypanosome and additional outgroup species.

Briefly, the phylogenetic analysis confirms the monophyly of the genus Trypanosoma with bootstrap support of $63 \%$ (Fig.). The human pathogenic trypanosomes, T. brucei and T. cruzi, are placed in separate clades, each receiving high bootstrap support of $>97 \%$. The brucei-clade, contains all species of mammalian Salivarian trypanosomes (Hoare 1972). Except for T. evansi and T. equiperdum, these trypanosomes are all of African origin and transmitted by tsetse flies [analysis of kinetoplast (mitochondrial) DNA (Borst et al. 1987) and isoenzymes (Lun et al. 1992, Gibson et al. 1983) points to $T$. evansi and T. equiperdum being comparatively recent mutants of $T$. brucei, which have been able to spread outside Africa because they no longer rely on tsetse transmission; the particularities of these two species are therefore irrelevant to the more ancient evolution of the clade]. The host exclusivity of this clade suggests a distinct evolutionary history initially confined to Africa. Trypanosomes of African origin from other vertebrates are completely unrelated (e.g. T. grayi, $T$. varani from African reptiles; T. mega from an African toad). A similar result is reported by Haag et al. (1998).

The cruzi-clade contains all subgenus Schizotrypanum species - T. cruzi isolates from humans, sylvatic and domestic mammals, including bats and opossums, together with trypanosomes specific to Old and New World bats, T. rangeli and an as yet unidentified trypanosome species from an Austra- lian kangaroo. The origins of parasites within this clade thus lie largely in South America and Australia; the only trypanosomes from this clade representing the Old World are those infecting bats.

The taxonomic and evolutionary status of human infective $T$. rangeli (generally classified as subgenus Herpetosoma) remains controversial (D'Alessandro \& Saravia 1992, Stevens \& Gibson 1998). In the current study $T$. rangeli, albeit only a single isolate (RGB - Basel), is classified firmly in a clade with a range of Schizotrypanum species (bootstrap 97\%); the classification of this isolate as $T$. rangeli is supported by preliminary results from analysis of the miniexon which indicate it to be of the correct size and sequence according to Murthy et al. (1992). T. rangeli and T. cruzi also cluster together (bootstrap >90\%) and separate from Salivarian trypanosomes in phylogenetic analyses of miniexon sequences (Stevens \& Gibson, unpublished data).

Phylogenetic resolution - Despite support for at least nine distinct clades within the Trypanosoma, it is not possible on the basis of these rRNA data to determine the exact order in which these clades diverged and a nine-way polytomy within the genus remains unresolved (Fig.). This may be due to limitations on the resolution of the ssu gene over this time scale, to a possibly explosive divergence of trypanosome species over a very short period some time around 100 million years before present (mybp) or, as seems probable, to a combination of both these factors.

Nevertheless, the inclusion of a large and varied range of taxa (Swofford et al. 1996) has enabled elucidation of the complex relationships of the human infective trypanosomes and, while saturation of some variable regions within the ssu gene may preclude the accurate determination of branching order at more ancient levels, considerable support for the 'correctness' of the phylogenetic relationships represented in the tree is provided by the logical placement of the outgroup trypanosomatids, Leishmania spp., Phytomonas spp. and Crithidia spp., which are well separated from the Trypanosoma (Fig.).

Comparative rates of sequence evolution - The phylogenetic analysis provides evidence of very different rates of evolution within the clades containing T. brucei and T. cruzi. Comparison of deeper branch lengths suggests a difference in intra-clade evolution rate of approximately 8 -fold. The exact extent to which the rapid evolution of certain lineages within the Salivarian clade may have distorted the topology of the tree (and hence estimates of evolutionary rates) is unknown. Nevertheless, the tree appears sufficiently robust to have avoided the Salivaria being drawn towards 
outgroup taxa by long-branch attraction (Felsenstein 1978, Hendy \& Penny 1989), a problem encountered in many previous studies (e.g. Fernandes et al. 1993, Maslov et al. 1994, 1996).

\section{DISCUSSION}

Phylogenetic analysis of variation in ssu rRNA genes of the 48 trypanosome specimens places the two human pathogens, T. brucei and T. cruzi, unequivocally in two distinct clades. The Asian primate trypanosome, $T$. cyclops, is grouped in an apparently unrelated clade with trypanosomes from cattle and deer.

The time of divergence of T. brucei and T. cruzi can be estimated by a range of methods including: sequence divergence analysis (the molecular clock approach), by reference to host phylogenies and by consideration of palaeo- and biogeographical data.

The concept of a molecular clock was first proposed by Zuckerkandl and Pauling (1965). Since then the exact nature of the workings of such 'clocks' have remained under almost constant debate (Sibley \& Ahlquist 1984, Wilson et al. 1987). It is apparent that, if they do exist, they are at best only stochastically constant (Fitch 1976), and that different types of DNA sequence undoubtedly evolve at significantly different rates. Nevertheless, within given taxonomic groups and defined categories of genetic marker, the concept of a molecular clock can provide a useful tool for understanding phylogenetic relationships.

In the study of trypanosome evolution molecular clocks have been used by a number of authors to attempt to date the divergence events between important taxonomic groups. In a recent and comprehensive study, Haag et al. (1998) used an estimate of $0.85 \%$ substitution per 100 million years, derived from rRNA based studies of Apicomplexa (Escalante \& Ayala 1995), to date the divergence of Salivarian trypanosomes from other trypanosomes at about 300 mybp. Such a result places the divergence of $T$. brucei and the Salivaria in the late Carboniferous, at a time when the very first reptiles had just appeared in a world dominated by amphibians, and long before the appearance of even the most primitive mammals. Perhaps significantly, the continents with which several major extant groups of trypanosomes (i.e. Africa: Salivaria; South America: Schizotrypanum) are now generally associated, had not at that time even begun to separate, but were grouped together in the solid southern land mass known as Gondwana (Cox \& Moore 1993, Smith et al. 1994).

A second method for estimating times of divergence in parasite phylogenies is based on congruence of host and parasite phylogenies, a much debated concept. Using this approach, parasite trees can be calibrated by reference to time points within host phylogenies, which have been constructed on the basis of independent evidence, e.g. fossils. Such an approach was also used by Haag et al. (1998), who used the divergence of fish from higher vertebrates (400 mybp) and the divergence of birds from rodents (220 mybp), to estimate the split of Salivarian trypanosomes from other trypanosomes at 260 and 500 mybp, respectively. Again, even the most recent of these estimates places the divergence of the Salivaria somewhat unrealistically in the mid-Permian.

A third approach to calibrating organismal phylogenies is by reference to known biogeographical events - vicariance biogeography (Nelson \& Rosen 1981) - and several sequence divergence based studies of trypanosomatids have drawn on this technique, for example, to date the divergence of Leishmania and Trypanosoma (Lake et al. 1988) and to date the split between Old and New World Leishmania (Nelson et al. 1990, Fernandes et al. 1993). Using this approach we previously obtained a mid-Cretaceous date for the divergence of $T$. brucei and T. cruzi (Stevens et al. 1998). In summary, we suggested that the exclusively African mammalian tsetse-transmitted taxon complement of the brucei-clade (excepting T. evansi and T. equiperdum - see above), points to an origin in Africa. The first time at which Africa became isolated was around 100 mybp, in the mid-Cretaceous, when it finally separated from South America and Euramerica (Parrish 1993, Smith et al. 1994). At this time, the first mammals were present, but had not yet begun major diversification and it is easy to envisage subsequent coevolution of this clade with ancient African hosts.

The cruzi-clade would thus have a southern super-continent (South American/Antarctica/Australia) origin, an interpretation which makes sense of the inclusion of the Australian marsupial trypanosome in the clade. Indeed, the early evolution of this clade may have been associated with the dominant marsupial fauna of the region. The opossum, Didelphis sp., a not so distant relative of the Australian kangaroos (Flannery 1989), is a particularly important natural reservoir of $T$. cruzi in South America and can maintain a patent parasitaemia throughout its life, with no apparent clinical symptoms (Deane et al. 1986). The only trypanosomes from this clade found in the Old World are those infecting bats. The biological similarity of T. cruzi and certain species of bat trypanosomes has been recognised for some time - all are classified in the subgenus Schizotrypanum. The present day distribution can be explained by the ability of bats to disperse over long distances, particularly across 
water barriers and, while $T$. (Schizotrypanum) species have been isolated from European bats on a number of occasions (Baker 1974, Baker \& Thompson 1971, Baker \& Mewis 1987), reports of $T$. cruzi-like trypanosomes from other hosts in the Old World are insubstantial (Hoare 1972).

In the current study, $T$. rangeli was placed firmly within the cruzi-clade. However, the taxonomic position of this human infective species has long been disputed. While it appears morphologically and behaviourally similar to subgenus Herpetosoma trypanosomes (Hoare 1972), it is transmitted by both salivarian and stercorarian routes (D'Alessandro \& Saravia 1992), while Añez (1982) even separated it into a new subgenus, Tejeraia. A limited study based on $\beta$-tubulin gene sequences (Amorim et al. 1993) suggested $T$. rangeli to be more closely related to $T$. brucei than to T. cruzi. While this result cannot be disputed, it is widely recognised (Swofford et al. 1996) that studies including limited numbers of taxa spanning disparate levels of relatedness are highly prone to artifactual effects. Certainly, the close relationship between $T$. rangeli and $T$. cruzi evident from ssu rRNA analysis has also been confirmed by comparison of miniexon sequences (Stevens \& Gibson, unpublished data). The miniexon sequence also confirms that the $T$. rangeli isolate used in the current study is a bona fide T. rangeli (Murthy et al. 1992).

The classification of T. cyclops with otherwise apparently unrelated $T$. (Megatrypanum) species and apart from other human/primate trypanosomes, suggests that its ability to infect primates has evolved independently (presumably in Asia) from species in either of the two clades containing human infective trypanosomes.

From the separate evolutionary histories of $T$. brucei and T. cruzi constructed from the phylogenetic evidence, we can deduce that their pathogenicity to humans developed on very different time scales. In Africa, T. brucei would have effectively co-evolved with hominids, since the first hominids evolved 5-15 mybp, the genus Homo 3 mybp (Johanson \& Taieb 1993) and Homo sapiens not earlier than 300000 years bp, presumably in continuous contact with both trypanosomes and tsetse flies. In contrast, human contact with $T$. cruzi would not have occurred prior to human migration into the Americas, which is generally dated no earlier than 30 - 40000 years bp. Moreover, there is no evidence for contact earlier than 3000 years bp when the first permanent settlements were made by previously nomadic cultures (Rothhammer et al. 1985). Humans, like other primates would have become infected as a simple addition to the already extensive host ranges of $T$. cruzi and T. rangeli (Hoare 1972).
Finally, to what extent their different evolutionary histories have affected intra-clade evolution rates is unknown, however, it appears that bruceiclade species are diverging at a rate up to eight times faster than that observed amongst cruzi-clade species. Moreover, such differences in evolutionary rates between trypanosome clades are in keeping with results from previous studies (Maslov et al. 1996) and are confirmed by maximum-likelihood based rate analyses (Stevens, Rambaut \& Gibson, unpublished data), which indicate the differences in rates of evolution between the Salivarian clade and the Schizotrypanum clade to be significant. Reasons for these rate differences remain to be explored.

\section{ACKNOWLEDGEMENTS}

To Chris Schofield for valuable discussions on the evolutionary relationships of $T$. cruzi.

\section{REFERENCES}

Amorim MI, Momen H, Traub-Cseko YM 1993. Trypanosoma rangeli: sequence analysis of $\beta$-tubulin gene suggests closer relationship to Trypanosoma brucei than to Trypanosoma cruzi. Acta Trop 53: 99-105.

Añez N 1982. Studies on Trypanosoma rangeli Tejera, 1920. IV. A reconsideration of its systematic position. Mem Inst Oswaldo Cruz 77: 405-415.

Baker JR 1963. Speculations on the evolution of the family Trypanosomatidae Doflein, 1901. Exp Parasitol 13: 219-233.

Baker JR 1974. Protozoan parasites of the blood of British wild birds and mammals. J Zool 172: 169-190.

Baker JR, Mewis GE 1987. Trypanosoma (Schizotrypanum) sp. indet. from a Maltese bat. Acta Trop 44: 99-100.

Baker JR, Thompson GB 1971. Two species of Trypanosoma from British bats. Trans $R$ Soc Trop Med Hyg 65: 427.

Borst P, Fase-Fowler F, Gibson WC 1987. Kinetoplast DNA of Trypanosoma evansi. Mol Biochem Parasitol 23: 31-38.

Cox CB, Moore PD 1993. Biogeography: An Ecological and Evolutionary Approach, 5th ed, Blackwell, Oxford, $326 \mathrm{pp}$.

D’Alessandro A, Saravia NG 1992. Trypanosoma rangeli, p. 1-54. In JP Kreier, JR Baker (eds), Parasitic Protozoa, 2nd ed, vol. 2, Academic Press, San Diego.

Deane MP, Lenzi HL, Jansen AM 1986. Double development cycle of Trypanosoma cruzi in the opossum. Parasitol Today 2: 146-147.

Escalante AA, Ayala FJ 1995. Evolutionary origin of Plasmodium and other Apicomplexa based on rRNA genes. Proc Natl Acad Sci USA 92: 5793-5797.

Felsenstein J 1978. Cases in which parsimony or compatibility will be positively misleading. Syst Zool 27: 401-410.

Fernandes AP, Nelson K, Beverley SM 1993. Evolution of nuclear ribosomal RNAs in kinetoplastid proto- 
zoa: Perspectives on the age and origins of parasitism. Proc Natl Acad Sci USA 90: 11608-11612.

Fitch WM 1976. Molecular evolutionary clocks, p. 160178. In FJ Ayala, Molecular Evolution, Sinauer, Sunderland, MA.

Flannery TF 1989. Phylogeny of the Macropodoidea; a study in convergence, p. 1-46. In G Grigg, P Jarman, I Hume (eds), Kangaroos, Wallabies and Rat-kangaroos, vol. 1, Surrey Beatty \& Sons, Chipping Norton, NSW.

Gibson WC, Wilson AJ, Moloo SK 1983. Characterisation of Trypanosoma (Trypanozoon) evansi from camels in Kenya using isoenzyme electrophoresis. Res Vet Sci 34: 114-118.

Haag J, O'Huigin C, Overath P 1998. The molecular phylogeny of trypanosomes: evidence for an early divergence of the Salivaria. Mol Biochem Parasitol 91: 37-49.

Hendy MD, Penny D 1989. A framework for the quantitative study of evolutionary trees. Syst Zool 38: 297-309.

Higgins DG, Bleasby AJ, Fuchs R 1992. Clustal V: improved software for multiple sequence alignment. Comp Applns Biosci 8: 189-191.

Hoare CA 1972. The Trypanosomes of Mammals, Blackwell, Oxford, 749 pp.

Johanson DC, Taieb M 1976. Plio-Pleistocene hominid discoveries in Hadar, Ethiopia. Nature 260: 293-297.

Johnson PD 1933. A case of infection by Trypanosoma lewisi in a child. Trans $R$ Soc Trop Med Hyg 26: 467.

Lake JA, De La Cruz VF, Ferreira PCG, Morel C, Simpson L. 1988. Evolution of parasitism: Kinetoplastid protozoan history reconstructed from mitochondrial rRNA gene sequences. Proc Natl Acad Sci USA 85: 4779-4783.

Lukes J, Jirku M, Dolezel D, Kral'ova I, Hollar L, Maslov DA 1997. Analysis of ribosomal RNA genes suggests that trypanosomes are monophyletic. J Mol Evol 44: 521-527.

Lun Z-R, Allingham R, Brun R, Lanham S 1992. The isoenzyme characteristics of Trypanosoma evansi and Trypanosoma equiperdum isolated from domestic stocks in China. Ann Trop Med Parasitol 86: 333-340.

Marché S, Roth C, Philippe H, Dollet M, Baltz T 1995. Characterization and detection of plant trypanosomatids by sequence analysis of the small subunit ribosomal RNA gene. Mol Biochem Parasitol 71: $15-26$.

Maslov DA, Avila HA, Lake JA, Simpson L 1994. Evolution of RNA editing in kinetoplastid protozoa. Nature 368: 345-348.

Maslov DA, Lukes J, Jirku M, Simpson L 1996. Phylogeny of trypanosomes as inferred from the small and large subunit rRNAs: implications for the evolution of parasitism in the trypanosomatid protozoa. Mol Biochem Parasitol 75: 197-205.

Meyers AA, Giller PS 1988. Analytical Biogeography, Chapman \& Hall, London, 578 pp.

Murthy VK, Dibbern KM, Campbell DA 1992. PCR amplification of mini-exon genes differentiates Try- panosoma cruzi from $T$. rangeli. Mol Cellular Probes 6: 237-243.

Neefs J-M, Van De Peer Y, Hendriks L, De Wachter R 1990. Compilation of small ribosomal subunit RNA sequences. Nucleic Acids Res 18: 2237-2243.

Nelson G, Rosen DE 1981. Vicariance Biogeography: A Critique, Columbia University Press, New York, $500 \mathrm{pp}$.

Nelson K, Alonso G, Langer PJ, Beverley SM 1990. Sequence of the dihydrofolate reductase-thymidylate synthase (DHFR-TS) gene of Leishmania amazonensis. Nucleic Acids Res 18: 2819.

Parrish JT 1993. The palaeogeography of the opening South Atlantic, p. 8-27. In W George, R Lavocat (eds), The Africa-South America Connection, Oxford Monographs on Biogeography No.7, Clarendon Press, Oxford.

Rothhammer F, Allison MI, Nunez L, Standen V, Arriaza B 1985. Chagas disease in pre-Colombian South America. Amer J Physical Anthropology 68: 495-498.

Sibley CG, Ahlquist JE 1984. The phylogeny of the hominoid primates, as indicated by DNA-DNA hybridization. J Mol Evol 20: 22-15.

Smith AG, Smith DG, Funnell BM 1994. Atlas of Mesozoic and Cenozoic Coastlines, Cambridge University, Cambridge, 99 pp.

Sogin ML, Elwood HJ, Gunderson JH 1986. Evolutionary diversity of eukaryotic small-subunit rRNA genes. Proc Natl Acad Sci USA 83: 1383-1387.

Stevens JR, Gibson WC 1998. The evolution of Salivarian trypanosomes. Second World Meeting on Salivarian Trypanosomes, Tryplink internet conference, EMBRAPA/ Centro de Pesquisa Agropecuaria do Pantanal, Brazil.

Stevens JR, Noyes HA, Dover GA, Gibson WC 1998. The ancient and divergent origins of the human pathogenic trypanosomes, Trypanosoma brucei and T. cruzi. Parasitology (in press).

Swofford DL, Olsen GJ, Waddell PJ, Hillis DM 1996. Phylogenetic inference, p. 407-514. In DM Hillis, C Moritz, BK Mable (eds), Molecular Systematics, Sinauer Associates, Sunderland, MA.

Vickerman K 1994. The evolutionary expansion of the trypanosomatid flagellates. Int J Parasitol 24: 1317 1331.

Weinman D 1972. Trypanosoma cyclops n. sp.: a pigmented trypanosome from the Malaysian primates Macaca nemestrina and $M$. ira. Trans $R$ Soc Trop Med Hyg 66: 628-636.

Wiemer EAC, Hannaert V, Van Den Ijssel PRLA, Van Roy J, Opperdoes FR, Michels PAM 1995. Molecular analysis of glyceraldehyde-3-phosphate dehydrogenase in Trypanosoma borelli: An evolutionary scenario of subcellular compartmentation in Kinetoplastida. J Mol Evol 40: 443-454.

Wilson AC, Ochman H, Prager EM 1987. Molecular time scale for evolution. Trends Genet 3: 241-247.

Zuckerkandl E, Pauling L 1965. Evolutionary divergence and convergence in proteins, p. 97-166. In V Bryson, HJ Vogel (eds), Evolving Genes and Proteins, Academic Press, New York. 